TRANSACTIONS OF THE AMERICAN MATHEMATICAL SOCIETY

Volume 348, Number 10, October 1996

\title{
EXTREMAL PROBLEMS AND SYMMETRIZATION FOR PLANE RING DOMAINS
}

\author{
A. YU. SOLYNIN AND M. VUORINEN
}

\begin{abstract}
We show that Teichmüller's classical lower bound for the capacity of a ring domain, obtained by circular symmetrization, can be replaced by an explicit one which is almost always better. The proof is based on a duplication formula for the solution of an associated extremal problem. Some inequalities are obtained for conformal invariants.
\end{abstract}

\section{INTRODUCTION}

Extremal problems for ring domains have been studied by many authors [T], [S], $[\mathrm{G}],[\mathrm{K}],[\mathrm{HLM}],[\mathrm{V} 2]$, and applications to geometric function theory are given in $[\mathrm{A}]$, $[\mathrm{LV}],[\mathrm{V} 1]$. An important extremal problem is that of finding lower bounds for the capacities of ring domains. O. Teichmüller $[\mathrm{T}]$ applied the circular symmetrization method to derive a sharp lower bound for this problem. We refer the reader to $[B],[D]$ for recent surveys of various applications of symmetrization techniques to function-theoretic problems.

The lower bounds given by the circular symmetrization method for the capacities of ring domains are sharp in a sense that will be explained below. We show in this paper that these estimates can, nevertheless, be replaced by new inequalities which are better than the symmetrization lower bounds except in the rare (symmetric) case when this lower bound already equals the value of the capacity. We are next going to formulate some of our main theorems and to give a brief survey of earlier results. For this purpose we first introduce some necessary notation.

For a pair $(E, F)$ of disjoint compact sets $E, F$ in $\overline{\mathbb{C}}=\overline{\mathbb{R}}^{2}$ (such a pair is usually called a condenser) let $\operatorname{cap}(E, F)$ denote the conformal capacity of $(E, F)$. For distinct points $a_{1}, a_{2}, a_{3}, a_{4} \in \overline{\mathbb{C}}$, let

$$
p\left(a_{1}, a_{2} ; a_{3}, a_{4}\right)=\inf _{E, F} \operatorname{cap}(E, F),
$$

where the infimum is taken over all pairs $(E, F)$ of disjoint continua with $a_{1}, a_{2} \in$ $E, a_{3}, a_{4} \in F$. For $z \in \mathbb{C} \backslash\{0,1\}$ let

$$
p(z)=p(0,1 ; z, \infty) .
$$

O. Teichmüller [T, p. 169] suggested the problem of finding the exact value of $p(z)$ in terms of well-known functions. M. Schiffer [S] solved the problem and showed that the extremal continua for the extremal problem (1.2) are images of linear sets under an elliptic function. Further results describing some properties of the extremal sets $E$ and $F$ in (1.2) appear in $[\mathrm{K}$, Ch. 5]. The conformal invariance of $\operatorname{cap}(E, F)$

Received by the editors November 18, 1994 and, in revised form, May 2, 1995.

1991 Mathematics Subject Classification. Primary 30C85; Secondary 31A15. 
implies that $p(z)$ depends only on $|z|$ and $|z-1|$. The circular symmetrization lower bound of Teichmüller yields the estimate

$$
p(z) \geq p(1+|z-1|)=\tau(|z-1|),
$$

where $\tau$ is an explicit function (cf. Section 2), with equality when $\operatorname{Im}\{z\}=$ $0, \operatorname{Re}\{z\}>1$. We shall show in Theorem 1.8 that an inequality better than (1.3) holds. Note that this better inequality also depends on $|z|$. It is shown in [LV, p. 57, Theorem 1.2] that inequality (1.3), together with the square root map, yields another inequality well-suited to certain situations. In our notation this result reads

$$
p(z) \geq \tau\left(A^{8}(z)\right), \quad A(z)=\left(s+\sqrt{s^{2}-1}\right)^{1 / 2}, \quad s=|z|+|z-1|,
$$

with equality if $\operatorname{Re}\{z\}=\frac{1}{2}$. We shall use the same notation $A(z)$ in the sequel.

We next show how information about the level curves of $p(z)$ would lead to immediate improvements of (1.3) and (1.4). Assume now that $z$ is fixed, $p(z)=c$, and that $x(z)$ is the point of intersection of the level curve $\{w \in \mathbb{C}: p(w)=c\}$ with the interval $(1, \infty)$. Then

$$
p(z)=p(x(z))=\tau(|x(z)-1|),
$$

because the points $z$ and $x(z)$ are both on the same level curve. Even though there is an explicit formula for $p(z)$ in [K, p. 192] (see Theorem 3.5 below), there is no well-known explicit formula for the distance $|x(z)-1|$ in terms of $|z|$ and $|z-1|$.

Although this problem about the level sets of $p(z)$ is unsolved, there are some explicit curves on which the values of $p(z)$ are monotone. We shall see below that some quadratic curves and the Cassini oval have this monotone property; we shall formulate the resulting explicit bounds for $p(z)$, which follow from the fact that we can give explicitly the point of intersection of these curves with the interval $(1, \infty)$.

Throughout this paper we use the following notation:

$$
I_{a}=\{z=x+i y: x>a, y>0\} .
$$

1.5. Theorem. (1) For $r \in(0, \infty), p\left(1+r e^{i \varphi}\right)$ is an increasing function of $\varphi$ on $(0, \pi)$.

(2) Let $L$ be an arc of an ellipse in $I_{1 / 2}$, with foci at 0 and 1 , or an arc of a hyperbola in $I_{1 / 2}$ with the same foci. If $z$ moves on $L$ so that $y$ increases, then $p(z)$ decreases. In particular, we have

$$
p(z) \leq \tau\left(\frac{1}{2}(|z|+|z-1|-1)\right)
$$

The monotone property in Theorem 1.5 (1), due to G. V. Kuz'mina [K, p. 206], easily implies (1.3). Theorem 1.5 (2) was proved in [SO1], where monotone properties on the arcs of a Euclidean or hyperbolic ellipse and hyperbola were established for two nonoverlapping domains on the Riemann sphere or in the hyperbolic plane.

The upper bound in Theorem 1.5 (2) blows up if $|z|+|z-1|=1$, while $p(z)<\infty$ for all $z \in \mathbb{C} \backslash\{0,1\}$. The next theorem provides a bound for $p(z)$ which is always finite and thus corrects this flaw in Theorem 1.5 (2).

1.6. Theorem. For all $z \in \mathbb{C} \backslash\{0,1\}$,

$$
p(z) \leq \tau\left(\frac{((\sqrt{|z|}+\sqrt{|z-1|}) A(z)-1)^{2}}{4(\sqrt{|z|}+\sqrt{|z-1|}) A(z)}\right) .
$$


One of our methods is the following functional identity for $p(z)$. Note that some of the above results could be combined with this formula to provide an alternative, possibly better, result. Our proof of this identity is geometric.

1.7. Theorem. For $z \in \mathbb{C} \backslash\{0,1\}, z \in I_{1 / 2}$, the following functional identity holds:

$$
p(z)=2 p\left(w^{4}\right), \quad w=\sqrt{z}+\sqrt{z-1} .
$$

Here the branches of the square roots are chosen so that $0<\arg \sqrt{z}<\pi / 2$ and $0<\arg \sqrt{z-1}<\pi$ when $z \in I_{1 / 2}$.

We shall refer to the functional identity in Theorem 1.7 as a duplication formula. We shall apply this duplication formula to prove Theorem 1.8 below.

We now define, for given $s>0$, the following two arcs of algebraic plane curves:

$$
\begin{gathered}
L(s)=\left\{w \in I_{1 / 2}: 2 A^{2}(w) \sqrt{|w||w-1|}=s\right\} \\
=\left\{w \in I_{1 / 2}: 4 \sqrt{|w||w-1|}\left|w-\frac{1}{2}+\sqrt{w(w-1)}\right|=s\right\}, \\
C O(s)=\left\{w \in I_{1 / 2}:|w||w-1|=s\right\} .
\end{gathered}
$$

The equivalence of these two definitions of $L(s)$ can be checked by routine calculations. Of course, $C O(s)$ is an arc of the Cassini oval.

1.8. Theorem. For given $z \in \mathbb{C} \backslash\{0,1\}$, define positive numbers

$$
t=2 \sqrt{|z||z-1|} A^{2}(z) \quad \text { and } \quad t_{1}=|z||z-1|
$$

so that $z \in L(t), \quad z \in C O\left(t_{1}\right)$. Then $p(z)$ decreases as $z$ moves on $L(t)$ or $C O\left(t_{1}\right)$ so that $\operatorname{Re}\{z\}$ increases. Furthermore, for $z \in \mathbb{C} \backslash\{0,1\}$,

$$
p(z) \geq p\left(1+x_{0}\right)=2 \tau(2 t) \geq \tau\left(x_{1}\right) \geq \tau(|z-1|),
$$

where $x_{0}=(1 / 2)(((1+t) / \sqrt{1+2 t})-1), x_{1}=(1 / 2)\left(\sqrt{1+4 t_{1}}-1\right)$. Next,

$$
p(z) \leq \tau\left(x_{2}\right)+4 / \tau\left(x_{2}\right), x_{2}=\frac{1+\sqrt{1-t^{2}}}{1-\sqrt{1-t^{2}}}
$$

for $|z| \leq 1 / 2$ or $|z-1| \leq 1 / 2$, and

$$
p(z) \leq 2 \tau(2 t-1)
$$

for $|z| \geq 1 / 2$ and $|z-1| \geq 1 / 2$. Equality occurs in (1) if $z=\operatorname{Re}\{z\}>1$ or $z=\operatorname{Re}\{z\}<0$, in (2) if $z=\operatorname{Re}\{z\} \in(0,1)$, and in (3) if $z=\operatorname{Re}\{z\}=1 / 2$.

The quadruple $0,1, z, \infty$ determines in a natural way the ring domains occurring in the definition of $p(z)=p(0,1 ; z, \infty)$. There are also "dual" ring domains associated with this same configuration of points, namely those separating $\{z, 1\}$ from $\{0, \infty\}$. This fact gives rise to an extremal problem, which in a certain sense is dual to the extremal problem of calculating $p(z)$. In the notation (1.1) the dual extremal quantity is $p(1, z ; 0, \infty)$. By conformal invariance we have $p(1, z ; 0, \infty)=p(z /(z-1))$. The next theorem, which provides a functional inequality for $p(z)$, studies these dual problems.

1.9. Theorem. For $z \in \mathbb{C} \backslash\{0,1\}, \operatorname{Re}\{z\} \geq 1 / 2$,

$$
4 \leq p(z) p\left(\frac{z}{z-1}\right) \leq 8 .
$$


Here equality holds in the first inequality if and only if $z \in(1, \infty)$, and in the second one if and only if $z=1 / 2$.

The paper concludes with a list of open problems.

\section{Preliminary Results AND SOME PRoOfs}

We shall use some basic facts of geometric function theory from [LV] and [V1]. In the unit disk $B=\{z:|z|<1\}$ of $\mathbb{C}$ the hyperbolic metric $\rho=\rho_{B}$ is defined by

$$
\text { th } \frac{\rho(a, b)}{2}=\frac{|a-b|}{\sqrt{|a-b|^{2}+\left(1-|a|^{2}\right)\left(1-|b|^{2}\right)}} .
$$

For the hyperbolic sine, cosine, tangent, and their inverses we use the notation sh, ch, th, arsh, arch, arth, respectively. In the hyperbolic half-plane $H=\{z \in \mathbb{C}$ : $\operatorname{Im}\{z\}>0\}$ the hyperbolic metric is given by

$$
\text { ch } \rho(a, b)=1+\frac{|a-b|^{2}}{2 \operatorname{Im}\{a\} \operatorname{Im}\{b\}} .
$$

A ring $D$ in $\mathbb{C}$ with nondegenerate complementary components can be mapped conformally onto $B \backslash E$, where $E$ is a compact subset of $B$. It can be shown [LV, p. 54], [V1, (5.56), (1.30)] that the modulus of the ring $D$ satisfies

$$
\bmod D \leq \mu\left(\operatorname{th} \frac{\rho(a, b)}{2}\right)
$$

for $a, b \in E$, with equality when $E$ is a geodesic arc of the hyperbolic metric with endpoints $a, b$ and where

$$
\mu(r)=\frac{\pi}{2} \frac{\mathcal{K}^{\prime}(r)}{\mathcal{K}(r)}, \quad \mathcal{K}(r)=\int_{0}^{1} \frac{d x}{\sqrt{\left(1-x^{2}\right)\left(1-r^{2} x^{2}\right)}}, \quad \mathcal{K}^{\prime}(r)=\mathcal{K}\left(\sqrt{1-r^{2}}\right),
$$

for $0<r<1$. The capacity of the ring $D$ is defined by

$$
\text { cap } D=2 \pi / \bmod D \text {. }
$$

Recall from the Introduction that if the boundary components of the ring $D$ are $E$ and $F$ then we also denote cap $D=\operatorname{cap}(E, F)$. For $t>0, R_{T}(t)$ is the Teichmüller ring with complementary components $[-1,0],[t, \infty]$; and $R_{G}(s)$ is the Grötzsch ring with complementary components $\bar{B}$ and $[s, \infty], s>1$. Then the capacities of these rings are given by

$$
\gamma(s)=\operatorname{cap} R_{G}(s)=2 \pi / \mu(1 / s), \tau(t)=\operatorname{cap} R_{T}(t)=\pi / \mu(1 / \sqrt{1+t}) .
$$

Both $\gamma$ and $\tau$ are strictly decreasing and

$$
\gamma(s)=2 \tau\left(s^{2}-1\right)=\tau\left(\frac{(s-1)^{2}}{4 s}\right) .
$$

We can rewrite (2.7) also as

$$
\gamma\left(\frac{1}{\operatorname{th} \frac{t}{2}}\right)=\tau\left(\frac{1}{e^{2 t}-1}\right)=2 \tau\left(\frac{2}{\operatorname{ch} t-1}\right), \quad t>0 .
$$

For more information about (2.1)-(2.8) see [LV] and [V1].

The connection between the hyperbolic metric and capacity is well known [TS]. To find inequalities between the hyperbolic metrics of two simply-connected domains we next introduce a convenient comparison function. Let $a, b \in \mathbb{C} \backslash\{0\}$ 
be distinct points and let $D \subset \mathbb{C}$ be a simply-connected domain with $0 \in \mathbb{C} \backslash D$. Then, by using Riemann's mapping theorem, one can define the hyperbolic metric $\rho(a, b)=\rho_{D}(a, b)$ for $a, b \in D$. For given $a, b \in \mathbb{C} \backslash\{0\}$ let

$$
r(a, b)=\inf \rho_{D}(a, b),
$$

where $D$ runs through all simply-connected domains in $\mathbb{C}$ with $0 \in \mathbb{C} \backslash D$.

There are equivalent definitions of the quantity $r(a, y)$ based on the use of capacities. Our first lemma summarizes the well-known estimates for $r(a, b)$.

2.10. Lemma. For $a, b \in \mathbb{C} \backslash\{0\}$

$$
\begin{aligned}
r(a, b) & \geq \frac{1}{2} \log \left(1+\frac{|a-b|}{\min \{|a|,|b|\}}\right), \\
\operatorname{th} \frac{r(a, b)}{2} & \geq \frac{|\sqrt{a}-\sqrt{b}|}{\sqrt{2(|a|+|b|)}}, \\
r(a, b) & \leq \frac{1}{2} \log \frac{|a|+|b|+|a-b|}{|a|+|b|-|a-b|}, \\
r(a, b) & \leq C_{0} \log \left(1+\frac{|a-b|}{\min \{|a|,|b|\}}\right),
\end{aligned}
$$

where $C_{0}=\log (3+2 \sqrt{2}) / \log 3 \approx 1.6045$.

Here bounds (1) and (3) coincide if $|a-b|=|| a|-| b||$, and thus these bounds are sharp in this case, whereas (2) is sharp if $|a|=|b|$ and (4) is sharp if $a=-b$.

Proof. We see that 2.10(1) and 2.10(3) follow from 1.5(1) and 1.5(2). Lemma 2.10(2) follows in turn from (2.3) and [LV, p. 51].

To prove (4) we may assume that $a=1, \quad b=1+\rho e^{i \beta},|b| \geq 1, \rho>0$. It follows from Theorem 1.5(1) that the function $r\left(1,1+\rho e^{i \beta}\right)$ is increasing in $\beta$ if $0 \leq \beta \leq \pi$. Thus it is sufficient to prove inequality (4) for $b=e^{i \theta}, \quad 0 \leq \theta \leq \pi$, and for $b \leq-1$.

In the first case we have

$$
r\left(1, e^{i \theta}\right)=\operatorname{arch}\left(1+2 \tan ^{2}(\theta / 4)\right) .
$$

Let

$$
\psi(\theta)=C_{0} \log (1+2 \sin (\theta / 2))-\operatorname{arch}\left(1+2 \tan ^{2}(\theta / 4)\right)
$$

Since

$$
\psi^{\prime \prime}(\theta)=-\frac{C_{0}}{2} \frac{2+\sin (\theta / 2)}{(1+2 \sin (\theta / 2))^{2}}-\frac{1}{8} \frac{\tan (\theta / 4)}{\cos (\theta / 4)}<0
$$

and

$$
\psi(0)=\psi(\pi)=0,
$$

we then obtain

$$
\psi(\theta)>0 \text { if } 0<\theta<\pi .
$$

In the second case let $b=-t, t>1$, and $D_{0}=\mathbb{C} \backslash[i 0, i \infty]$. Then

$$
r(1,-t) \leq \rho_{D_{0}}(1,-t) .
$$


Mapping the domain $D_{0}$ onto the half-plane $H$ and using (2.2) we obtain

$$
\operatorname{ch} \rho_{D_{0}}(1,-t)=1+\frac{1+t}{\sqrt{t}} .
$$

From (2.11) and (2.12) it follows that it is sufficient to prove

$$
\varphi_{1}(\tau) \geq \varphi_{2}(\tau)
$$

where $\varphi_{1}(\tau)=\operatorname{ch}\left(C_{0} \log \tau\right), \quad \varphi_{2}(\tau)=1+\frac{\tau-1}{\sqrt{\tau-2}}, \quad \tau \geq 3$. Since

$$
\varphi_{1}^{\prime \prime}(\tau)=C_{0} \tau^{-2}\left(C_{0} \operatorname{ch}\left(C_{0} \log \tau\right)-\operatorname{sh}\left(C_{0} \log \tau\right)\right)>0,
$$

we conclude that

$$
\varphi_{1}^{\prime}(\tau) \geq \varphi_{1}^{\prime}(3)=\left(C_{0} / 3\right) \operatorname{sh}\left(C_{0} \log 3\right) \approx 1.5128 .
$$

These inequalities, together with the easily verified relation

$$
\varphi_{2}(\tau)=\frac{\tau-3}{2(\tau-2)^{3 / 2}}<\varphi_{2}(5)=3^{-3 / 2} \approx 0.1925,
$$

yield (2.13).

Even the exact value of $r(a, b)$ is known; see (3.4) and Theorem 3.5 below. Since this exact value is quite complicated it is desirable to use more concrete inequalities such as Lemma 2.10 above. We next prove other similar results that improve Lemma 2.10. The majorant in Lemma 2.10(3) blows up if $|a|+|b|=|a-b|$, while Theorem 2.14 below always yields a finite bound.

2.14. Theorem. For $a, b \in \mathbb{C} \backslash\{0\}$ there exists a ray $F$ with $0 \in F, a, b \in \mathbb{C} \backslash F$ such that, with $D=\mathbb{C} \backslash F$,

$$
\operatorname{ch} \rho_{D}(a, b)=1+\frac{|a|+|b|-\sqrt{(|a|+|b|)^{2}-|a-b|^{2}}}{\sqrt{|a||b|}+\frac{1}{2} \sqrt{(|a|+|b|)^{2}-|a-b|^{2}}} .
$$

In particular, for $a, b \in \mathbb{C} \backslash\{0\}$,

$$
\text { ch } r(a, b) \leq 1+\frac{|a|+|b|-\sqrt{(|a|+|b|)^{2}-|a-b|^{2}}}{\sqrt{|a||b|}+\frac{1}{2} \sqrt{(|a|+|b|)^{2}-|a-b|^{2}}} .
$$

Here equality holds if $|a-b|=|| a|-| b||$ or if $|a|=|b|$.

Proof. We shall construct a ring with complementary components $F$ and $E$ as follows. Let $e$ be a unit vector such that $[0, e]$ bisects the smaller angle between the segments $[0, a]$ and $[0, b]$, and let $F=\{-s e: s \geq 0\}$. We may assume that $e=-1$. Then map $\mathbb{C} \backslash F$ by $\sqrt{z}$ onto $H$, and join $\sqrt{a}, \sqrt{b}$ by a geodesic segment $E_{1}$ of the hyperbolic metric of $H$. Then $E_{1}$ is an arc of a circle perpendicular to $\overline{\mathbb{R}}=\partial H$. Let $E$ be the image of $E_{1}$ under $z^{2}$ joining $a$ to $b$. With $D=\mathbb{C} \backslash F$ we have $\rho_{D}(a, b)=\rho_{H}(\sqrt{a}, \sqrt{b})$, while $(2.2)$ yields

$$
\operatorname{ch} \rho_{H}(\sqrt{a}, \sqrt{b})=1+\frac{|\sqrt{a}-\sqrt{b}|^{2}}{2 \operatorname{Im}\{\sqrt{a}\} \operatorname{Im}\{\sqrt{b}\}} .
$$

If $\alpha \in(0, \pi)$ is the angle between $[0, a]$ and $[0, b]$, then $\frac{\alpha}{2}$ is the angle between $\sqrt{a}$ and $\sqrt{b}$, and $\operatorname{Im}\{\sqrt{a}\}=\sqrt{|a|} \cos \frac{\alpha}{4}$. The Law of Cosines yields

$$
\cos ^{2} \frac{\alpha}{2}=\frac{(|a|+|b|)^{2}-|a-b|^{2}}{4|a||b|},
$$


EXTREMAL PROBLEMS AND SYMMETRIZATION FOR PLANE RING DOMAINS 4101

$$
|\sqrt{a}-\sqrt{b}|^{2}=|a|+|b|-2 \sqrt{|a||b|} \cos \frac{\alpha}{2}=|a|+|b|-\sqrt{(|a|+|b|)^{2}-|a-b|^{2}} .
$$

These, together with (2.15) and the definition (2.8), yield the desired relations. The equality statements follow if we use the formulas for the modulus of Teichmüller's and Mori's rings [LV, p. 56]. 口

2.16. Proof of Theorem 1.6. Choose disjoint continua $E$ and $F$ in $\overline{\mathbb{C}}$ such that $0,1 \in$ $E, z, \infty \in F$, and such that $D=\overline{\mathbb{C}} \backslash E$ is simply connected. Then, by the Riemann Mapping Theorem,

$$
p(z) \leq 2 \pi / \mu\left(\operatorname{th} \frac{\rho_{D}(z, \infty)}{2}\right)=\gamma\left(1 / \operatorname{th} \frac{\rho}{2}\right),
$$

where $\rho_{D}=\rho$ is the Poincaré metric of $D$. By (2.8),

$$
\gamma\left(1 / \operatorname{th} \frac{\rho}{2}\right)=2 \tau(2 /(\operatorname{ch} \rho-1)) \text {. }
$$

Next, in view of Theorem 2.14 , we can choose the sets $E, F$ above such that with $s=|z|+|z-1|, \quad g=\sqrt{|z||z-1|}$, we have

$$
\operatorname{ch} \rho=1+\frac{s-\sqrt{s^{2}-1^{2}}}{g+\frac{1}{2} \sqrt{s^{2}-1^{2}}}
$$

Combining these relations we obtain

$$
\begin{aligned}
p(z) & \leq 2 \tau\left(\frac{2 g+\sqrt{s^{2}-1}}{s-\sqrt{s^{2}-1}}\right)=2 \tau\left(A^{2}(z)\left(2 g+\sqrt{s^{2}-1}\right)\right) \\
& =2 \tau\left(A^{2}(z)(2 g+s)-1\right)=\tau\left(\frac{(A(z) \sqrt{2 g+s}-1)^{2}}{4 A(z) \sqrt{2 g+s}}\right),
\end{aligned}
$$

where in the last step $(2.7)$ was used. Noting that $2 g+s=(\sqrt{|z|}+\sqrt{|z-1|})^{2}$ we see that the desired conclusion follows. Applying the results of this section we now prove some corollaries. $\square$

2.17. Corollary. Let $f: B \rightarrow \mathbb{C}$ be $K$-quasiconformal and zero-free in $B$. Then, for $\left|z_{1}\right|<1, \quad\left|z_{2}\right|<1$,

$$
\frac{\mid \sqrt{f\left(z_{1}\right)}-\sqrt{f\left(z_{2}\right) \mid}}{\sqrt{2\left(\left|f\left(z_{1}\right)\right|+\left|f\left(z_{2}\right)\right|\right)}} \leq \varphi_{K}\left(\frac{\left|z_{1}-z_{2}\right|}{\sqrt{\left|z_{1}-z_{2}\right|^{2}+\left(1-\left|z_{1}\right|^{2}\right)\left(1-\left|z_{2}\right|^{2}\right)}}\right),
$$

where $\varphi_{K}(r)=\mu^{-1}(\mu(r) / K)$.

Proof. There exists a continuum $F$ joining the points 0 and $\infty$ such that $f(z)$ maps $B$ onto the domain $D=\mathbb{C} \backslash F$. It follows from (2.9) and $[\mathrm{LV}, \mathrm{p}$. 65] that

$$
\operatorname{th} \frac{r\left(f\left(z_{1}\right), f\left(z_{2}\right)\right)}{2} \leq \operatorname{th} \frac{\rho_{D}\left(f\left(z_{1}\right), f\left(z_{2}\right)\right)}{2} \leq \varphi_{K}\left(\operatorname{th} \frac{\rho\left(z_{1}, z_{2}\right)}{2}\right) .
$$

After this observation, the result follows from (2.1) and 2.10 (2).

Our new bound for $p(z)$ and the method of proving Theorem 4.1 in [V2] lead immediately to the following refinement of this theorem from [V2].

2.18. Corollary. Let $f: \mathbb{C} \backslash E \rightarrow \mathbb{C} \backslash B$ be a $K$-quasiconformal mapping, where $E$ is a connected set with $0,1 \in E$, and let $f(\infty)=\infty$. Then, for $z \in \mathbb{C} \backslash E$,

$$
|f(z)| \leq \gamma^{-1}\left(\frac{1}{K} \gamma(\sqrt{1+2 t})\right) \leq 4^{K-1}(1+2 t)^{K / 2},
$$

where $t$ is defined in Theorem 1.8 . 
A look at the proof of Theorem 2.11 shows that, in fact, the following result holds.

2.19. Corollary. For given $z_{0} \in \mathbb{C} \backslash\{0,1\}$ there exists an arc $E$ joining 0 and 1 with $z_{0} \notin E$ such that the conformal mapping $f$ of $G=\mathbb{C} \backslash E$ onto $\mathbb{C} \backslash \bar{B}$ satisfies

$$
\left|f\left(z_{0}\right)\right|=\left(\sqrt{\left|z_{0}\right|}+\sqrt{\left|z_{0}-1\right|}\right) A\left(z_{0}\right),
$$

where $A\left(z_{0}\right)$ is as in Theorem 1.6. In the cases $\operatorname{Im}\left\{z_{0}\right\}=0, \operatorname{Re}\left\{z_{0}\right\}>1$ or $\operatorname{Re}\left\{z_{0}\right\}<0, \quad z_{0}=\frac{1}{2}+i t, t \neq 0$, the arc $E$ is unique.

Proof. Let $F$ be the ray defined in Theorem 2.14 for points $z_{0}$ and $z_{0}-1$ in the $w$-plane, and let $E$ be the image of $F$ under the mapping $w \mapsto z_{0} /\left(z_{0}-w\right)$. Then $E$ is a circular arc joining 0 and 1 . If $D=\mathbb{C} \backslash F, G=\overline{\mathbb{C}} \backslash E$, and $f$ is a conformal mapping of $G$ onto $\overline{\mathbb{C}} \backslash \bar{B}$ with $f(\infty)=\infty$ then, by conformal invariance of the hyperbolic metric,

$$
\operatorname{ch} \rho_{\mathbb{C} \backslash F}\left(z_{0}, z_{0}-1\right)=\operatorname{ch} \rho_{\overline{\mathbb{C}} \backslash E}\left(z_{0}, \infty\right)=\operatorname{ch} \rho_{\overline{\mathbb{C}} \backslash \bar{B}}\left(f\left(z_{0}\right), \infty\right) .
$$

These relations, together with Theorem 2.14 , imply the equality

$$
\frac{\left|z_{0}\right|+\left|z_{0}-1\right|-A(z)}{\sqrt{\left|z_{0}\right|\left|z_{0}-1\right|}+\frac{1}{2} A(z)}=\frac{1}{\left|f\left(z_{0}\right)\right|^{2}-1}
$$

which yields the assertion after some calculation.

Uniqueness of the arc $E$ follows from the fact that the ray $F$ is an $\operatorname{arc}$ of a hyperbolic geodesic in the cases under consideration. $\square$

2.20. Remark. We shall see below in Example 2.21 that the quantity $r(x, y)$ is not a metric. We remark in passing that a related question from [V1, p. 193] was recently solved in the affirmative independently by J. A. Jenkins [J2] and A. Yu. Solynin [SO2]. They proved that $\lambda_{\mathbb{C} \backslash\{0\}}(x, y)^{-1}$ is a metric. Jenkins has also extended this result to any plane domain with finite connectivity. J. Ferrand $[\mathrm{F}]$ has proved a more general result for Riemannian manifolds.

2.21. Example. We next show that

$$
r(-1,1)>r(-1, i)+r(i, 1)=2 r(i, 1) .
$$

Using the case of equality in Theorem 2.14, we see that

$$
\begin{gathered}
\operatorname{ch} r(-1,1)=3, \operatorname{ch} r(-1, i)=\operatorname{ch} r(i, 1)=7-4 \sqrt{2}, \\
3>\operatorname{ch}(2 \operatorname{arch}(7-4 \sqrt{2}))=161-112 \sqrt{2} \approx 2.60 .
\end{gathered}
$$

The last line verifies the asserted inequality.

\section{The Duplication Formula AND COMPUtation of The MODUli}

A notation slightly different from $p(z)$ is used in $[\mathrm{K}]$. There, for complex $a \in$ $\mathbb{C} \backslash\{-1,1\}$, she defines

$$
\log M(a)=\sup _{D} \bmod (D)
$$

where $D$ runs through the collection of all ring domains separating $\{-1,1\}$ and $\{a, \infty\}$. Let $h$ be the similarity map with $h(H)=H$ and $h(-1)=0, h(1)=1$. Then $h(z)=(z+1) / 2$, and

$$
p(h(a))=p((a+1) / 2)=2 \pi / \log M(a)
$$


by conformal invariance of the modulus. Conversely, for $z \in \mathbb{C} \backslash\{0,1\}$,

$$
p(z)=2 \pi / \log M\left(h^{-1}(z)\right)=2 \pi / \log M(2 z-1),
$$

so that $p(z)$ and $\log M(a)$ are equivalent. To find a relation between $\log M(a)$ and $r(c, d)$, we map $a$ to 0 by $z \mapsto z-a$. Then $-1 \mapsto-1-a, 1 \mapsto 1-a$, and we get

$$
\begin{aligned}
\gamma\left(\frac{1}{\operatorname{th} \frac{r(1-a,-1-a)}{2}}\right) & =\gamma\left(\frac{1}{\operatorname{th} \frac{r(a+1, a-1)}{2}}\right)=\frac{2 \pi}{\log M(a)} \\
& =\frac{2 \pi}{\mu\left(\operatorname{th} \frac{r(a+1, a-1)}{2}\right)} .
\end{aligned}
$$

Thus

$$
\log M(a)=\mu\left(\operatorname{th} \frac{r(a+1, a-1)}{2}\right) .
$$

This is the first part of the exact formula for $r(z, w)$ mentioned in Section 2 . The second part is the explicit formula for $\log M(a)$ given in the following theorem, which goes back to H. Grötzsch, O. Teichmüller, and M. Schiffer, and which in its present form appears in [K, p. 192].

3.5. Theorem. For $a \in \mathbb{C} \backslash\{-1,1\}$ the following formula holds:

$$
\log M(a)=\pi \operatorname{Im}\left\{i \frac{\mathcal{K}^{\prime}(r)}{\mathcal{K}(r)}\right\}, r^{2}=\frac{2}{1+a} .
$$

Here the elliptic integrals $\mathcal{K}(r)$ and $\mathcal{K}^{\prime}(r)$ are understood to be positive for $r^{2} \in$ $(0,1)$ with the explicit formula (2.4), defined for $\operatorname{Im}\left\{r^{2}\right\} \neq 0$ by analytic continuation along any path not intersecting the real axis of the $r^{2}$-plane, and defined for $\operatorname{Im}\left\{r^{2}\right\}=0$ and $r^{2} \notin[0,1]$ by analytic continuation along any path in the lower half-plane $\operatorname{Im}\left\{r^{2}\right\} \leq 0$.

In the case $a \notin[-1,1]$ the maximum in (3.1) is realized only for the domain $D(a)$ obtained from the complex plane $\mathbb{C}$ by making cuts along the closures of the critical trajectories of the quadratic differential

$$
Q(z, a) d z^{2}=e^{i \beta(a)}\left[\left(z^{2}-1\right)(z-a)\right]^{-1} d z^{2},
$$

where

$$
\beta(a)=-\arg r^{2} \mathcal{K}^{2}(r),
$$

while in the case $-1<a<1$ it is realized only by the domain $D(a)$ and the domain symmetric to it with respect to the real axis.

3.7. Remark. It follows from Theorem 3.5 that $\log M(a)$ is a harmonic function in the upper half plane.

The explicit formula for $\log M(a)$ in Theorem 3.5 is rather difficult to use for practical calculations. It would be preferable to have a formula in terms of the distances $|a-1|$ and $|a+1|$ or in terms of the angles of the triangle with vertices $-1,1, a$. We next obtain an expression for $\log M(a)$, as a limit of a certain sequence, which is more suitable for calculations.

3.8. Proof of Theorem 1.7. We first prove a duplication formula for $\log M(a)$, which then easily gives the desired formula for $p(z)$. 
Let $a \in \bar{I}_{0}, a \neq 1$, and let $D(a)$ be the extremal domain in the sense of Theorem 3.5. Let $\tilde{D}(a)$ denote a domain obtained from $D(a)$ by adding a boundary continuum joining the points $a$ and $\infty$. By $G(a)$ we denote the image of the set $\tilde{D}(a)$ under the double-valued mapping

$$
\zeta=2 \frac{\sqrt{1-a}+i \sqrt{1+a}}{\sqrt{1-a}-i \sqrt{1+a}} \frac{\sqrt{z-a}-i \sqrt{1+a}}{\sqrt{z-a}+i \sqrt{1+a}}-1 .
$$

Here the branches of the square roots $\sqrt{1 \pm a}$ are chosen so that $0 \leq \arg \sqrt{1 \pm a}<\pi$.

The set $G(a)$ is a ring separating the pairs of points $\{-1,1\}$ and $\{F(a), \infty\}$, where

$$
F(a)=2\left(a+\sqrt{a^{2}-1}\right)^{2}-1
$$

and $0<\arg \sqrt{a^{2}-1}<\pi / 2$ if $a \in I_{0}$.

The moduli of the rings $G(a)$ and $D(a)$ are connected by the relation

$$
\bmod (G(a))=2 \bmod (D(a)) .
$$

We claim that $G(a)$ is extremal in the sense of Theorem 3.5. For if not, then there would exist an extremal $\operatorname{ring} G^{\prime}$ such that $\bmod \left(G^{\prime}\right)>\bmod (G(a))$.

Let $G^{\prime \prime}$ be the image of $G^{\prime}$ under the Möbius transformation

$$
z_{1}=i \sqrt{1+a} \frac{2(\sqrt{1-a}+i \sqrt{1+a})+(\zeta+1)(\sqrt{1-a}-i \sqrt{1+a})}{2(\sqrt{1-a}+i \sqrt{1+a})-(\zeta+1)(\sqrt{1-a}-i \sqrt{1+a})},
$$

and let $L=\left\{z_{1}:\left|g\left(z_{1}\right)\right|=|g(0)|\right\}$ be a level curve of a function $g\left(z_{1}\right)$ which maps the ring $G^{\prime \prime}$ conformally onto a suitable circular annulus. Clearly, $G^{\prime \prime}$ is the only ring with maximum modulus among all rings separating the pair of points $\sqrt{1-a}, i \sqrt{1+a}$ from the pair $-\sqrt{1-a},-i \sqrt{1+a}$. These pairs correspond to each other under the transformation $z_{1} \mapsto-z_{1}$. Therefore, by the uniqueness of the extremal ring, the domain $G^{\prime \prime}$ and the curve $L$ are invariant under this transformation. The latter imply that $L$ divides $G^{\prime \prime}$ into two rings, say $G^{(1)}$ and $G^{(2)}$, such that

$$
\bmod \left(G^{(1)}\right)=\bmod \left(G^{(2)}\right)=(1 / 2) \bmod \left(G^{\prime}\right) .
$$

In addition, the function $\zeta=z_{1}^{2}+a$ is univalent in each of the domains $G^{(1)}$ and $G^{(2)}$. Let $D^{\prime}$ be the image of $G^{(1)}$ under the mapping $\zeta=z_{1}^{2}+a$. The ring $D^{\prime}$ separates the points -1 and 1 from $a$ and $\infty$ and satisfies the relations

$$
\bmod \left(D^{\prime}\right)=\bmod \left(G^{(1)}\right)>(1 / 2) \bmod (G(a))=\bmod (D(a)),
$$

contradicting the extremality of the ring $D(a)$.

Hence $G(a)$ has maximum modulus among all rings separating the points -1 and 1 from the points $F(a)$ and $\infty$.

Rewriting the relation between the moduli of $G(a)$ and $D(a)$ in an equivalent form we get the following identity, which holds for all $a \in \bar{I}_{0} \backslash\{1\}$ :

$$
\log M(a)=(1 / 2) \log M(F(a)) .
$$

Formula (3.10) is the "duplication formula" for $\log M(a)$. The proof now follows from (3.3) and (3.10).

The simple method applied above for doubling the modulus of a ring is well known and was used, for instance, by O. Lehto and K. I. Virtanen to prove formula (1.4) (cf. Theorem 1.2, Ch. II [LV]). 
In what follows we shall need some properties of the mapping produced by the function $\zeta=F(z)$, where $F(z)$ is defined by (3.9). At first we define some curves. Let $G_{0}=H \backslash\{z:|z+1| \leq 2\}$, and for $t>0$ let

$$
\mathcal{L}(t) \equiv\{z \in H: \log M(z)=t\}
$$

be arcs of the level curve of $\log M(z)$ lying in the upper half-plane $H$; let $\mathcal{L}_{+}(t)=$ $\mathcal{L}(t) \cap \bar{I}_{0}, \mathcal{L}^{\prime}(t)=\mathcal{L}(t) \cap \bar{G}_{0}$.

From Remark 3.7 and Theorem 1.5 (1) it follows easily that $\mathcal{L}(t)$ is symmetric with respect to the imaginary axis; it consists of one analytic arc if $t \geq \pi / 2$ or two if $0<t<\pi / 2$. Moreover, $\mathcal{L}(t)$ has at most one point of intersection with each of the circles $\mathbb{T}_{r}( \pm 1), r>0$, where $\mathbb{T}_{r}\left(z_{0}\right) \equiv\left\{z:\left|z-z_{0}\right|=r\right\}$.

For $t>0$ let

$$
l(t)=\left\{z \in \bar{I}_{0}: \sqrt{\left|z^{2}-1\right|}\left(|z+1|+|z-1|+\sqrt{(|z+1|+|z-1|)^{2}-4}\right)=2 t\right\} .
$$

It is clear that the arc $l(t)$ is the image, under the linear transformation $z \mapsto 2 z-1$ of the arc $L(t)$ introduced in Section 1 .

Let

$$
E(t)=\left\{z \in \bar{I}_{0}:|z+1|+|z-1|=2(1+t)\right\}, \quad t>0,
$$

be an arc in $I_{0}$ of an ellipse with foci \pm 1 .

3.12. Lemma. (1) The function $\zeta=F(z)$ gives the conformal mapping of $I_{0}$ onto the domain $G_{0}$ so that the intervals $[1,+\infty]$ and $[i 0,+i \infty]$ correspond to the intervals $[1,+\infty]$ and $[-3,-\infty]$ and the interval $(0,1)$ corresponds to the semicircle $\mathbb{T}_{2}^{+}(-1)$, where $\mathbb{T}_{r}^{+}(a)=\mathbb{T}_{r}(a) \cap H$.

(2) The image under this mapping of the arc $\mathcal{L}_{+}(t)$ is the arc $\mathcal{L}^{\prime}(2 t)$ and the images of the arcs $l(t)$ and $E(t)$ are, respectively, the arcs of the circles $\mathbb{T}_{r_{1}}(1)$ and $\mathbb{T}_{r_{2}}(-1)$ lying in $G_{0}$, with

$$
r_{1}=4 t, \quad r_{2}=2(1+t+\sqrt{t(t+2)})^{2} .
$$

(3) The following inequalities hold:

$$
\begin{array}{cll}
\arg F(a)>\arg a & \text { for } & a \in \bar{I}_{0} \backslash[1, \infty], \\
|F(a)|>|a| & \text { for } & a \in \bar{I}_{0} \backslash\{1\} .
\end{array}
$$

Proof. The mapping $\zeta=F(z)$ is a composition of the Joukowski inverse mapping $z \mapsto z+\sqrt{z^{2}-1}$, the mapping $z \mapsto z^{2}$, and the linear mapping $z \mapsto 2 z-1$. These immediately yield (1) and the statement about the arc $E(t)$. The statement about the arc $\mathcal{L}_{+}(t)$ follows from the duplication formula $(3.10)$.

To prove the assertion about the arc $l(t)$ let us consider the preimage $l_{1}(t)$ of the arc of $\mathbb{T}_{r_{1}}(1)$ under the mapping $\zeta=F(z)$. For $z \in l_{1}(t)$ we have

$$
\left|\left(z+\sqrt{z^{2}-1}\right)^{2}-1\right|=2 t .
$$

The last relation gives one of the equivalent definitions of the arc $l(t)$ (cf. Introduction).

By direct calculation one can prove that the inequalities (3) with $>$ replaced by $\geq$ are true on the boundary of the quadrant $I_{0}$. Hence, by the maximum principle for harmonic functions, (3) follows. ㅁ 
3.13. Proof of Theorem 1.8. Lemma 3.12, Theorem 1.5 (1), and Theorem 1.7 imply the monotone statement of Theorem 1.8 concerning the curve $L(t)$. (They also lead to a new proof of the statement in Theorem $1.5(2)$ about the monotoneity on an ellipse.) Calculating the values of $p(z)$ at the end points of $L(t)$ we obtain the first inequality in (1) and inequalities (2), (3).

To prove the monotoneity of $p(z)$ on the Cassini oval, consider $\operatorname{arcs} l_{1}, l_{2}$, and $l_{3}$ of the curves $\mathbb{T}_{r}(1), C O\left(t_{1}\right)$, and $L(t)$, which go into the half-plane $\left\{\operatorname{Re}\{z\}>\operatorname{Re}\left\{z_{0}\right\}\right\}$ from the point $z_{0} \in I_{1 / 2}$. It is not difficult to see that $|z||z-1|$ increases as $z$ moves on $l_{1}$ starting from $z_{0}$. Also the quantity $2 A^{2}(z) \sqrt{|z||z-1|}$ (see the definition of $L(t))$ increases as $z$ moves on $l_{2}$. Therefore, in a neighborhood of $z_{0}$, the arc $l_{2}$ lies between $l_{1}$ and $l_{3}$.

By Theorem 3.5 (see Remark 3.7) the level curves of the function $p(z)$ are analytic. Noting that the angle formed by $l_{1}$ and $l_{3}$ is less than $\pi$ we see easily that $p(z)$ increases in a neighborhood of $z_{0}$ as $z$ moves on an arbitrary smooth arc (for instance on the Cassini oval) starting from $z_{0}$ and lying between $l_{1}$ and $l_{3}$.

Calculating a value of $p(z)$ at the right end of $C O\left(t_{1}\right)$, we obtain the second inequality in (1). ㅁ

Now we apply the duplication formula to express the function $\log M(a)$ as a limit of a simple recursive sequence.

Let $a \in \bar{I}_{0} \backslash\{1\}, a_{0}=a$. If the complex number $a_{n-1} \in \bar{I}_{0} \backslash\{1\}$ has been defined, then the number $a_{n}$ is defined by

$$
a_{n}= \begin{cases}\tilde{a}_{n} & \text { if } \operatorname{Re} \tilde{a}_{n} \geq 0, \\ -\overline{\tilde{a}}_{n} & \text { if } \operatorname{Re} \tilde{a}_{n}<0,\end{cases}
$$

where $\tilde{a}_{n}=F\left(a_{n-1}\right)$. It follows easily from Lemma $3.12(3)$ that

$$
a_{n} \rightarrow \infty \quad \text { as } \quad n \rightarrow \infty
$$

Using the duplication formula, we obtain the relation

$$
\log M(a)=2^{-n} \log M\left(a_{n}\right), \quad n=0,1,2, \ldots .
$$

By Theorem 1.5 (1) and (3.15) we have

$$
2^{-n} \log M\left(\left|a_{n}-1\right|-1\right) \leq \log M(a) \leq 2^{-n} \log M\left(\left|a_{n}-1\right|+1\right) .
$$

For $a>1$, relations (3.4), (2.6), and (2.8) yield

$$
\log M(a)=2 \mu(\sqrt{2 /(a+1)}) .
$$

Applying (3.16), (3.17), and the relation

$$
\mu(r)=\log \left(\frac{4}{r}-r-\delta_{1}(r)\right), \quad 0<r<1,
$$

with $r^{3} / 4<\delta_{1}(r)<2 r^{3}$, obtained by O. Lehto and K. I. Virtanen [LV, p. 62], we deduce the relation

$$
\log M(a)=2^{-n}\left\{\log \left|a_{n}\right|+\log 8+O\left(\left|a_{n}\right|^{-1}\right)\right\},
$$

which gives a simple and convenient method for calculation of the function $\log M(a)$.

Using the duplication formula and Lemma 3.12, we shall construct the sequence of points $b_{n}$ on the same level curve of $\log M(a)$ such that $b_{n} \rightarrow x(a)$, where $x(a)$ is the point of intersection of the mentioned level curve and the interval $(1,+\infty)$. 
We shall denote by $F^{[n]}$ and $F^{[-n]}$ the $n$th iterates of the functions $F(z)$ and $F^{-1}(z)$, respectively, if these iterations are defined, $n=1,2, \ldots$

It follows from the first inequality of Lemma 3.12 (3) that for each complex number $a \in \bar{I}_{0} \backslash[1, \infty]$ there exists a natural number $N=N(a)$ such that

$$
a_{N}=F^{[N]}(a)
$$

is well-defined and $\operatorname{Re}\left\{a_{N}\right\}<0$.

Each of the level curves of $\log M(a)$ intersects the semicircle $\mathbb{T}_{2}^{+}(-1)$ at most once. Hence Lemma 3.12 (2) implies the existence of the number $b \in \bar{I}_{0}$ such that

$$
b=F^{[-N(a)]}\left(-\bar{a}_{N(a)}\right) .
$$

Using the duplication formula we obtain the equality

$$
\log M(b)=\log M(a)
$$

and the fact that $b$ lies on the corresponding level curve of $\log M(a)$ between $a$ and the point of intersection of this level curve with the positive real axis $\mathbb{R}_{+}$.

Let $b_{0}=a, b_{1}=b$. If $b_{1} \in(1, \infty)$, then we have found the desired point. If this is not the case, then we can use the described procedure to construct the points

$$
b_{k}=F^{\left[-N\left(b_{k-1}\right)\right]}\left(-\overline{F^{\left[N\left(b_{k-1}\right)\right]}}\left(b_{k-1}\right)\right), \quad k=2, \ldots, J,
$$

such that

$$
\log M\left(b_{k}\right)=\log M(a),
$$

where $J$ is a natural number or $\infty$, and $x(a)=b_{J} \in(1, \infty)$ if $J<\infty$; or $b_{k} \rightarrow x(a)$, where $x(a) \in(1, \infty)$ and $\log M(x(a))=\log M(a)$, if $J=\infty$.

3.20. Some particular cases. In some particular cases the calculation of the function $\log M(a)$ can be simplified further. $\operatorname{In}$ case $\operatorname{Im}\{a\}=0, \operatorname{Re}\{a\}>1$, the extremal ring in the definition of $\log M(a)$ is similar to the Teichmüller ring, and another simple particular case occurs when $\operatorname{Re}\{a\}=0$. In the latter case the extremal domain is Mori's ring, and $\log M(a)$ is equal to the modulus of this Mori's ring. These particular cases are well known $[\mathrm{LV}],[\mathrm{K}]$.

A nontrivial particular case where $\log M(a)$ can be evaluated in simplified form occurs for $-1<a<1$. Indeed, Theorem 3.5 and the following formulas of the table 4 of [Bat, p. 319] (note that we have the sign "-" in our first formula instead of "+" in the corresponding formula of [Bat] because we use the analytic continuation along paths in the lower half-plane instead of along paths in the upper half-plane as in $[$ Bat $]$ )

$$
\begin{aligned}
\mathcal{K}(1 / \kappa) & =\kappa\left[\mathcal{K}(\kappa)-i \mathcal{K}^{\prime}(\kappa)\right], \\
\mathcal{K}^{\prime}(1 / \kappa) & =\kappa \mathcal{K}^{\prime}(\kappa),
\end{aligned}
$$

imply, for $-1<a<1$ that

$$
\log M(a)=\frac{\pi \mathcal{K}(s) \mathcal{K}^{\prime}(s)}{\mathcal{K}^{2}(s)+\mathcal{K}^{\prime 2}(s)},
$$

or

$$
\log M(a)=\left(\pi^{2} / 2\right)\left(\mu(s)+\mu\left(s^{\prime}\right)\right)^{-1},
$$




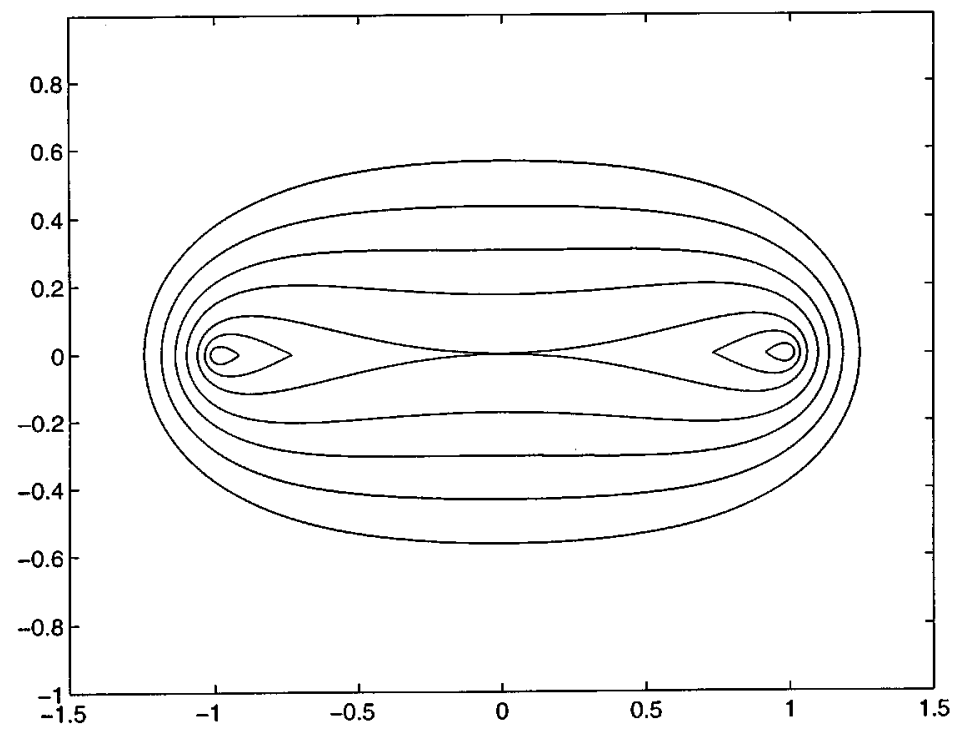

FiguRE 3.1. Some level curves of $\log M(a)$

where $s^{2}=\frac{1}{2}(1-|a|), s^{\prime}=\sqrt{1-s^{2}}$. Note that, by formula (3.21), $\log M(a)$ is a decreasing function of $a$ for $a \in(0,1)$. Using the duplication formula and (3.21) one can derive an expression for $\log M(a)$ in certain cases similar to (3.21). For instance, for $a= \pm 1+2 e^{i \theta}$ we obtain

$$
\begin{aligned}
\log M\left(-1+2 e^{i \theta}\right) & =2 \log M(\cos (\theta / 2)) \\
& =\frac{2 \pi \mathcal{K}(\sin (\theta / 4)) \mathcal{K}(\cos (\theta / 4))}{\mathcal{K}^{2}(\sin (\theta / 4))+\mathcal{K}^{2}(\cos (\theta / 4))} .
\end{aligned}
$$

Numerical values of complete elliptic integrals of complex arguments have been tabulated in $[\mathrm{FC}]$.

Finally, we point out the following relations (see [SO2]):

$$
\begin{aligned}
& \log M(1+\varepsilon)=-\frac{\pi^{2}}{\log |\varepsilon|}\left(1+\frac{5 \log 2}{\log |\varepsilon|}\right)+o\left(\log ^{-2}|\varepsilon|\right), \\
& (\log M(1+\varepsilon))^{-1}=-\pi^{-2}(\log |\varepsilon|-5 \log 2)+\alpha(\varepsilon),
\end{aligned}
$$

where $\alpha(\varepsilon) \rightarrow 0$ if $\varepsilon \rightarrow 0$. Some level curves of the function $\log M(A)$ are shown in Figure 3.1.

\section{Dual problems for Ring Domains}

For the proof of Theorem 1.9 we need the following two results for general plane rings. In the case of rings having complementary components on a Jordan curve the first (without the equality statement) is proved in [GS, p. 715].

4.1. Theorem. Let $D_{1}, D_{2}$ be rings in $\overline{\mathbb{C}}$, and let $E_{1}^{(k)}$ and $E_{2}^{(k)}$ be the complementary continua of $D_{k}, k=1,2$. If $E_{m}^{(k)} \cap E_{n}^{(l)} \neq \emptyset, k, l, m, n=1,2$ and $k \neq l$, 
then

$$
\operatorname{cap}\left(D_{1}\right) \cdot \operatorname{cap}\left(D_{2}\right) \geq 4
$$

Equality holds here only for rings $\varphi\left(D_{1}(t)\right)$ and $\varphi\left(D_{2}(t)\right)$, where $D_{1}(t)=$ $((\overline{\mathbb{C}} \backslash[-1,1]) \backslash[-\infty,-1 / t]) \backslash[1 / t, \infty], D_{2}(t)=(\overline{\mathbb{C}} \backslash[-1 / t,-1]) \backslash[1,1 / t], t>1$, and $\varphi$ is a Möbius transformation.

Proof. The set $D_{1} \backslash E_{1}^{(2)} \backslash E_{2}^{(2)}$ contains at least two quadrilaterals $Q^{\prime}$ and $Q^{\prime \prime}$, each of which has one pair of distinguished sides on the continua $E_{1}^{(2)}$ and $E_{2}^{(2)}$ and a second pair on the continua $E_{1}^{(1)}$ and $E_{2}^{(1)}$. By Theorem 3 [SO3] we have

$$
\bmod D_{1} \leq(1 / 4)\left(\bmod Q^{\prime}+\bmod Q^{\prime \prime}\right) \text {. }
$$

Again equality in (4.2) occurs if and only if the set $D_{1} \backslash Q^{\prime} \backslash Q^{\prime \prime}$ consists of two arcs $\gamma_{1}$ and $\gamma_{2}$ that correspond to opposite radial segments under a conformal mapping of $D_{1}$ onto the circular annulus.

Using the Grötzsch Lemma (see e.g. [J1, p. 23]) we obtain the inequality

$$
\bmod \left(Q^{\prime}\right)+\bmod \left(Q^{\prime \prime}\right) \leq 1 / \bmod \left(D_{2}\right) .
$$

Equality in (4.3) occurs if and only if the arcs $\gamma_{1}$ and $\gamma_{2}$ are the complementary continua of the domain $D_{2}$ and the set $D_{2} \backslash Q^{\prime} \backslash Q^{\prime \prime}$ consists of arcs $\gamma_{3}$ and $\gamma_{4}$, which correspond to opposite radial segments under the conformal mapping of $D_{2}$ onto the circular annulus. The desired inequality follows from (4.2) and (4.3).

If equality holds in Theorem 4.1 then it holds in (4.2) and (4.3) as well. Let $f_{k}$ map the ring $D_{k}$ onto a suitable domain $D_{k}(t)$ so that $f_{k}\left(Q^{\prime}\right)=H, k=1,2$. It is easy to show that $f_{1}=f_{2}$ on the set $Q^{\prime} \cup Q^{\prime \prime}$. Hence $f_{1}$ can be continued to an automorphism of $\overline{\mathbb{C}}$. $\square$

4.4. Theorem. Let $\gamma_{1}$ and $\gamma_{2}$ be two intersecting closed curves on $\overline{\mathbb{C}}$, let the points $z_{1}^{(l)}, z_{2}^{(l)}$ divide the curve $\gamma_{l}$ into the arcs $\gamma_{l}^{\prime}$ and $\gamma_{l}^{\prime \prime}$, and let the points be separated by the curve $\gamma_{s}, l, s=1,2, s \neq l$. Then

$$
\operatorname{cap}\left(\gamma_{1}^{\prime}, \gamma_{2}^{\prime \prime}\right) \cdot \operatorname{cap}\left(\gamma_{1}^{\prime \prime}, \gamma_{2}^{\prime}\right) \geq 16
$$

Equality holds here if and only if the curves $\gamma_{1}$ and $\gamma_{2}$ are orthogonal circles on $\overline{\mathbb{C}}$ and the points $z_{1}^{(l)}$ and $z_{2}^{(l)}$ are symmetric to each other with respect to $\gamma_{s}, l, s=$ $1,2, s \neq l$.

We omit the proof of this theorem, because it is similar to the proof of Theorem 4.1 .

4.5. Proof of Theorem 1.9. By definition we have $p(z)=p(0,1 ; z, \infty)$ and $p\left(\frac{z}{z-1}\right)=$ $p(1, z ; 0, \infty)$. Hence the first inequality in Theorem 1.9 follows from Theorem 4.1.

Using Theorem 1.5 (1) we see that both factors of the product $p(z) p\left(\frac{z}{z-1}\right)$ increase when $z$ moves in the upper half plane on the arc of the circle $\{w:|w-1|=t\}$ in the positive direction. We consider separately the two cases $t \in(0,1 / 2]$ and $t>$ $1 / 2$. In the first case let $a=1-t$ and in the second let $a=\frac{1}{2}+i h, h=\sqrt{t^{2}-1 / 4}$. With this notation, in both cases we have $p(z) p\left(\frac{z}{z-1}\right) \leq p(a) p\left(\frac{a}{a-1}\right)$.

Case 1. $\frac{1}{2} \leq a<1$. Let $G_{a}=\mathbb{C} \backslash\{z=a+i t: 0 \leq t<\infty\}$ and $D_{a}=G_{a} \backslash E$, where $E$ is the hyperbolic geodesic segment joining the points 0 and 1 in $G_{a}$. It is clear that

$$
p(a) \leq \operatorname{cap}\left(D_{a}\right)
$$


We claim that

$$
\operatorname{cap}\left(D_{a}\right) \cdot p\left(\frac{a}{a-1}\right) \leq 8
$$

Using the equality statement in Theorem 4.1, we obtain

$$
p\left(\frac{a}{a-1}\right)=4 / p(1 / a) .
$$

Hence (4.6) is equivalent to the inequality

$$
\operatorname{cap}\left(D_{a}\right) \leq 2 \tau\left(\frac{1-a}{a}\right)
$$

or, in view of (2.8), to the inequalities

$$
\begin{gathered}
\operatorname{cap}\left(D_{a}\right) \leq \gamma\left(a^{-1 / 2}\right), \\
\operatorname{ch} \rho_{G_{a}}(0,1) \leq \operatorname{ch} \rho\left(0, a^{1 / 2}\right) .
\end{gathered}
$$

By using (2.2) and simple calculation we obtain the inequality

$$
(a(1-a))^{-1 / 2} \leq 2 a(1-a)^{-1},
$$

which obviously holds if $\frac{1}{2} \leq a<1$. Hence (4.7) holds which, together with (4.6), proves Theorem 1.8 in the case $\frac{1}{2} \leq a<1$.

Case 2. $a=\frac{1}{2}+i h, h>0$. The function

$$
\zeta=(1-\sqrt{z}) /(1+\sqrt{z})
$$

maps the domain $D_{0}=\overline{\mathbb{C}} \backslash[-\infty, 0]$ onto the unit disk $B$. Moreover, $\zeta(1)=0$ and $\left|\zeta\left(\frac{1}{2}+i h\right)\right|=r_{1}$, where

$$
\begin{gathered}
r_{1}^{2}=\left(1-2^{3 / 2} \sqrt{\cos \alpha} \cos (\alpha / 2)+2 \cos \alpha\right) /\left(1+2^{3 / 2} \sqrt{\cos \alpha} \cos (\alpha / 2)+2 \cos \alpha\right), \\
\alpha=\arctan 2 h .
\end{gathered}
$$

Let $G(h)=\overline{\mathbb{C}} \backslash\left[\frac{1}{2}+i h, \frac{1}{2}-i \infty\right] \backslash\left\{z=\frac{1}{2}+i h+\rho e^{i \theta},-\alpha \leq \theta \leq \pi+\alpha\right\}, \rho=$ $\sqrt{(1 / 4)+h^{2}}$. In the case considered here the extremal domain connected with the problem of $p(a)$ is Mori's ring. Therefore, by the equality statement of Theorem 4.4 , we have the relation

$$
p(a)=16 / \operatorname{cap}(G(h)) .
$$

This last relation shows that the assertion of the theorem, in Case 2, will follow from the inequality

$$
\gamma\left(r_{1}^{-1}\right) \leq(1 / 2) \operatorname{cap}(G(h))
$$

The function

$$
\zeta=i(w-i) /(w+i),
$$

where $w=\sqrt{i(z-a)} / \sqrt[4]{(1 / 4)+h^{2}}$, maps $G(h)$ onto the domain $U \backslash\left[-r_{2}, r_{2}\right]$, where

$$
r_{2}^{2}=(\sqrt{2}+\sin (\alpha / 2)-\cos (\alpha / 2)) /(\sqrt{2}-\sin (\alpha / 2)+\cos (\alpha / 2)) .
$$

Hence

$$
(1 / 2) \operatorname{cap}(G(h))=\gamma\left(r_{2}^{-2}\right) .
$$


Thus (4.8) is equivalent to the inequality

$$
r_{1}^{2} \leq r_{2}^{4}
$$

or

$$
\frac{1-2^{3 / 2} \sqrt{\cos \alpha} \cos (\alpha / 2)+2 \cos \alpha}{1+2^{3 / 2} \sqrt{\cos \alpha} \cos (\alpha / 2)+2 \cos \alpha} \leq \frac{3+2^{3 / 2}(\sin (\alpha / 2)-\cos (\alpha / 2))-\sin \alpha}{3-2^{3 / 2}(\sin (\alpha / 2)-\cos (\alpha / 2))-\sin \alpha} .
$$

Replacing $\sqrt{\cos \alpha}$ by $\cos \alpha$ one can see, after some calculations, that the latter inequality will follow from

$$
3 \cos \alpha \cos (\alpha / 2)-\frac{1}{2} \sin (2 \alpha) \cdot \cos (\alpha / 2)+(1+2 \cos \alpha)(\sin (\alpha / 2)-\cos (\alpha / 2)) \geq 0 .
$$

Multiplying by $\cos (\alpha / 2)$ we obtain the equivalent inequality

$$
\sin (2 \alpha)(1-\cos \alpha)+2(\sin \alpha)(1-\sin \alpha) \geq 0,
$$

which is clearly correct if $0 \leq \alpha \leq \pi / 2$. This completes the proof. $\square$

4.9. Open problems. (1) Find a generalization of Theorem 1.7 to the $n$-dimensional case. Some results in this case were obtained in [V2].

(2) Extend the results of Section 4 to the $n$-dimensional case.

Acknowledgments. This work was supported in part by the Academy of Finland and the Finnish Ministry of Foreign Affairs. We are indebted to the referee and to Professor G. D. Anderson for their helpful remarks on an earlier version of this paper.

\section{REFERENCES}

[A] L. V. Ahlfors, Lectures on Quasiconformal Mappings, Van Nostrand, 1966. MR 34:336

[B] A. Baernstein II, Some topics in symmetrization, Harmonic Analysis and Partial Differential Equations, ed. by J. Garcia-Cuerra, Lecture Notes in Math. 1384 (1989), 111-123. MR 90h: 26022

[Bat] Bateman Manuscript Project, Higher Transcendental Functions (A. Erdelyi, ed.), vol. 2, 1955. MR 16:586c

[D] V. N. Dubinin, Symmetrization in geometric theory of functions (Russian), Uspehi Mat. Nauk 49 (1994), 3-76. CMP 95:05

[F] J. Ferrand, Conformal capacities and extremal metrics, Manuscript, January 1994.

[FC] H. E. Fettis and J. C. Caslin, A Table of the Complete Elliptic Integral of the First Kind for Complex Values of the Modulus, Wright-Patterson Air Force Base, Ohio, Aerospace Research Laboratories, Office of Aerospace Research, U.S. Air Force, 1969. MR 40:6725

[GS] F. P. Gardiner and D. P. Sullivan, Symmetric structures on a closed curve, Amer. J. Math. 114 (1992), 683-736. CMP 92:16

[G] F. W. Gehring, Symmetrization of rings in space, Trans. Amer. Math. Soc. 101 (1961), 499-519. MR 24:A2677

[HLM] D. A. Herron, Xianyang Liu, and D. Minda, Ring domains with separating circles or separating annuli, J. Analyse Math. 53 (1989), 233-252. MR 91i:30018

[J1] J. Jenkins, Univalent functions and conformal mapping, Ergebnisse der Math. Vol. 18, Corrected ed., Springer-Verlag, Berlin-Heidelberg-New York, 1965.

[J2] J. Jenkins, On metrics defined by modules, Pacific J. Math. (to appear).

[K] G. V. Kuz'mina, Moduli of Families of Curves and Quadratic Differentials, Proc. Steklov Institute of Math., 1982, Issue 1 (Russian original: Tom 139, 1980). MR 84j:30038b

[LV] O. Lehto and K. I. Virtanen, Quasiconformal Mappings in the Plane, Die Grundlehren der math. Wissenschaften, 2nd ed., vol. 126, Springer-Verlag, Berlin-Heidelberg-New York, 1973. MR 49:9202

[S] M. Schiffer, On the modulus of doubly-connected domains, Quart. J. Math. Oxford Ser. 17 (1946), 197-213. MR 8:325b 
[SO1] A. Yu. Solynin, On the extremal decompositions of the plane or disk on two nonoverlapping domains (Russian), Kuban University, Krasnodar, 1984, Deponirovano in VINITI, N7800, $16 \mathrm{p}$.

[SO2] A. Yu. Solynin, Moduli of doubly connected domains and conformally invariant metrics (Russian), Zap. Nautchn. Semin. LOMI, Tom 196, 1991, pp. (122-131). MR 93e:30016

[SO3] A. Yu. Solynin, The solution of an isoperimetric problem of Polya-Szegö (Russian), vol. 168, Zap. Nauchn. Sem. LOMI, 1988, pp. 140-154. MR 90h:30059

[T] O. Teichmüller, Untersuchungen über konforme und quasikonforme Abbildung, Deutsche Math. 3 (1938), 621-678.

[TS] M. Tsuji, Potential Theory in Modern Function Theory, 2nd ed., Chelsea Publishing Co., New York, 1975. MR 54:2990

[V1] M. Vuorinen, Conformal Geometry and Quasiregular Mappings, Lecture Notes in Math., vol. 1319, Springer-Verlag, 1988. MR 89k:30021

[V2] M. Vuorinen, Conformally Invariant Extremal Problems and Quasiconformal Mappings, Quarterly J. Math. Oxford (2) 43 (1992), 501-514. MR 93i:30020

Steklov Institute, Fontanka 27, St. Petersburg 191011, Russia

E-mail address: solynin@pdmi.ras.ru

FAX : 007-812-3105377

University of Helsinki, FiN-00100 Helsinki, Finland

E-mail address: vuorinen@csc.fi

FAX: 358-0-1913213 